\title{
Resultados Perinatais de Gêmeos com Pesos Discordantes ao Nascer
}

\author{
Perinatal Results of Birth Weight-Discordant Twins \\ Eduardo Martins Marques ${ }^{1}$, Marilza Vieira Cunha Rudge ${ }^{2}$
}

\begin{abstract}
RESUMO
Objetivo: estudar as influências da diferença de pesos entre gêmeos, no nascimento, sobre o resultado perinatal.

Métodos: analisaram-se, retrospectivamente, as informações referentes aos partos gemelares ocorridos na Maternidade do Hospital Regional de Clinicas de Sorocaba, SP, de julho de 1997 a junho de 1998. A amostragem foi composta de 89 mães e seus gêmeos, divididos em três classes de diferença de pesos ao nascer: com concordância (diferença <15\%), discordância leve (de 15 a 25\%) e discordância grave (>25\%). As variáveis independentes analisadas foram essas três classes e as dependentes foram: baixo peso ao nascer, indice de Apgar menor que 7 no primeiro e quinto minuto, nascimentos pré-termo, tempo médio de internação do recém-nascido no berçário e coeficiente de mortalidade perinatal I. Para análise estatística utilizaram-se o teste de Kruskal-Wallis, complementado pelo teste de Hollander, e o teste de Blackwell.

Resultados: a incidência de discordância de pesos entre pares de gêmeos foi de 30,3\%, sendo 19,1\% de discordância leve e 11,2\% de discordância grave. Observamos nas classes, respectivamente, os números de gestações (62, 17 e 10) e de nascimentos pré-termo (32, 9 e 7). Para o primeiro e o segundo gêmeo, observamos: baixo peso ao nascer (39/41, 13/12 e 8/9), Apgar $<7$ no primeiro minuto (16/13, 3/ 7 e 2/3), Apgar menor que 7 no quinto minuto (4/4, 0/2 e 1/2), tempo médio (dias) de internação no berçário (3,7/3,7, 4,6/6,0 e 7,3/8,7) e coeficiente de mortalidade perinatal I (22,4/16,8, 0/16,8 e 5,6/5,6).

Conclusões: o baixo peso ao nascer e nascimentos pré-termo foram mais freqüentes nos gêmeos da classe com discordância grave. Houve tendência ao agravamento progressivo do resultado perinatal, respectivamente, nas classes com concordância, discordância leve e discordância grave.
\end{abstract}

PALAVRAS-CHAVE: Gemelidade. Gêmeos discordantes. Morbidade e mortalidade perinatal.

\section{Introdução}

As gestações múltiplas, consideradas como doenças obstétricas, têm, na opinião de Baldwin ${ }^{1}$, cinco aspectos que devem ser considerados: há doenças que são mais freqüentes nos gêmeos, conseqüentes ao fato de dois indivíduos ocuparem o espaço onde usualmente só existe um: prematuridade,

${ }^{1}$ Disciplina de Obstetrícia, Faculdade de Ciências Médicas de Sorocaba (CCMB/PUC-SP).

${ }^{2}$ Disciplina de Obstetrícia, Faculdade de Medicina de Botucatu (UNESP).

Maternidade do Hospital Regional de Clínicas de Sorocaba, SP.

Correspondência:

Eduardo Martins Marques

R. Capitão Manoel Januário, 84 - V. Amélia

18051-390 - Sorocaba - SP

Fone/fax: (15) 224-1744

e-mail: edmama@terra.com.br problemas da parturição e discordância de pesos ao nascer. Há doenças que são exclusivas dos gêmeos, como a sindrome da transfusão feto-fetal enquanto certas enfermidades podem ter sua expressão modificada pela gemelidade (as discordâncias nas manifestações dos grupos sangüineos, com incompatibilidade em gêmeos dizigóticos e as variações nas manifestações de doenças metabólicas ou infecciosas decorrentes de anastomoses vasculares ou da localização intra-uterina). De descrição mais recente, alguns trabalhos sugerem que o processo ou estímulo para gemelidade pode afetar o surgimento de neoplasia e leucemia. Finalmente, há alguns aspectos sociais, como a dificuldade para criar os gêmeos, que pode contribuir para o abuso ou a negligência pré e pós-natal ${ }^{1}$.

O baixo peso ao nascer na gemelidade pode decorrer da prematuridade, do menor peso para a idade gestacional e da discordância de pesos en- 
tre os pares de gêmeos. A sua importância decorre da maior incidência, quando comparada aos recém-nascidos provenientes de gestação única, e da maior freqüência de mortalidade ${ }^{1}$. O baixo peso para a idade gestacional é aceito como sendo melhor parâmetro para predizer o mau resultado perinatal, do que a discordância de pesos e, nos gêmeos, é responsável por 50 a $85 \%$ de toda mortalidade perinatal ${ }^{2}$. A falha ou a diminuição no crescimento pode afetar um ou ambos os gêmeos e alguns dos exemplos mais notáveis de baixo peso ao nascer estão associados com as discrepâncias ponderais da gemelidade ${ }^{1}$.

Na literatura sobre gêmeos, são evidentes a falta de consenso na padronização de intervalos de diferenças de pesos e o correspondente desfecho perinatal ${ }^{3}$. Tais intervalos são arbitrários e nem sempre os investigadores apuraram associação entre eles e os prognósticos perinatal e neonatal ${ }^{4}$. A maioria dos trabalhos procura a correlação mais precisa, entretanto, ainda não existe uma definição consolidada.

Os resultados de mortalidade perinatal e neonatal, admissão de unidades de terapia intensiva, taxa de anomalias congênitas e índice de Apgar no quinto minuto foram associados a diversas categorias de diferenças de pesos entre gêmeos. Foram significantes quando se compararam os grupos com menos de $15 \%$ e mais de $30 \%$ de diferença de pesos para: índice de Apgar no quinto minuto $(9,5 \%$ contra $16,7 \%$ ), recém-nascidos com necessidade de cuidados intensivos $(8,8 \%$ contra $41,7 \%)$ e mortalidade perinatal $(1,5 \% \text { contra } 33 \%)^{5-8}$.

Nas gestações monocoriônicas, os fetos estão muito mais passiveis de morrerem, no periodo perinatal, se comparados àqueles de gestações simples ${ }^{9}$. O risco de morte perinatal é 2,5 vezes maior, quando há diferença de pesos menor que $25 \%$, e o risco de morte fetal é 6,5 vezes maior, quando os pesos diferem em $25 \%$ ou mais ${ }^{10}$. Gêmeos pré-termo, com diferenças de pesos superiores a $30 \%$, apresentaram altas taxas de morbidade: malformações congênitas $(37,5 \%)$, recém-nascidos pequenos para a idade gestacional $(31,8 \%)$, indice de Apgar no quinto minuto menor que $7(33,3 \%)$ e leucomalácia periventricular $(16,7 \%)^{4}$. Foram relatados também valores mais baixos do índice de Apgar no quinto minuto no gêmeo de menor peso, bem como períodos mais longos de internação neonatal, independentemente da via de parto ${ }^{6}$.

Além das questões relacionadas à diferença de peso, os gêmeos discordantes com peso inferior a $2.000 \mathrm{~g}$ têm risco dez vezes maior de apresentar resultados adversos, logo após o parto ${ }^{11}$. A sindrome da angústia respiratória foi mais freqüente em gêmeos discordantes que nos concordantes, e no menor gêmeo, nas discordâncias superiores a
$15 \%{ }^{11}$. A morbidade está aumentada no segundo gêmeo, com relação ao primeiro, quando ele é o menor deles ${ }^{10}$. Assim, não só a discordância de pesos, mas, também, a ordem do nascimento do gêmeo menor seria relevante para o prognóstico ${ }^{10}$.

A idade gestacional no parto não está relacionada ao grau de diferença de pesos ${ }^{6}$. Os partos ocorrem mais freqüentemente entre 37 e 38 semanas de gestação ${ }^{10}$ e 1,4 semana antes, nos pares discordantes $^{11}$. Esses gêmeos tornam-se indivíduos com dificuldade adaptativa na vida adulta, por conta do risco perinatal aumentado e do desenvolvimento físico e intelectual menos favorável ${ }^{12}$.

Estudando o arquivo de partos da Maternidade do Conjunto Hospitalar de Sorocaba, notamos existir grande número de nascimentos de gêmeos com pesos distintos e que eles apresentaram muitas intercorrências clínicas. Esses fatos nos levaram a indagar sobre a correlação entre o grau de diferença de peso e o resultado perinatal nesses gêmeos; assim como sobre o melhor critério para identificar os intervalos de diferença de pesos, que seriam relevantes. Pretendemos, nesse estudo diagnosticar os pares de gêmeos sob risco e verificar o resultado perinatal.

\section{Pacientes e Métodos}

Elaboramos estudo clínico-observacional e retrospectivo, com base em protocolo preestabelecido para levantar dados registrados, em prontuários, de mulheres com gravidez múltipla e de seus recém-nascidos. Foram analisados os prontuários de mulheres com gestação múltipla e de seus recém-nascidos (RN) atendidas no período de um ano, entre julho de 1997 e junho de 1998, na Maternidade do Hospital Regional de Clínicas de Sorocaba (HRCS), São Paulo. Esse é um hospital universitário, que atende a uma população estimada em 1.800.000 habitantes.

Foram consideradas elegiveis para participar do trabalho grávidas com idade gestacional de mais de 20 semanas, com parto gemelar ocorrido no HRCS, no período do estudo. Foram analisados 101 prontuários e selecionados os de 89 mulheres com parto gemelar e seus $178 \mathrm{RN}$. Foram excluídos 12 prontuários: quatro referentes a internações clínicas cujos partos ocorreram fora do HRCS, cinco de partos trigemelares e três de abortamentos gemelares.

As variáveis independentes estudadas foram as classes de diferença de pesos entre gêmeos: concordância, discordância leve e discordância grave. A variável dependente estudada foi o resultado perinatal, avaliado por: baixo peso ao nascer, indice de Apgar menor que 7 no primeiro e 
quinto minutos, incidência de nascimentos prétermo, tempo médio de internação dos RN no berçário e coeficiente de mortalidade perinatal I. As variáveis de controle estudadas foram: a idade materna, a multiparidade (dois ou mais partos) e o tipo de parto.

Os RN foram identificados pela ordem do nascimento em primeiro e segundo gêmeo e pesados, logo após o nascimento, em balança pesabebê marca Filizola ${ }^{\circledR}$, modelo B.P. baby $2057 / 97$, com precisão de $5 \mathrm{~g}$. As classes de diferença de pesos foram obtidas pela relação: (peso do maior gêmeo - peso do menor gêmeo)/peso do maior gêmeo x $100^{5,7}$.

De acordo com a classe de diferença de pesos, os RN foram classificados em gêmeos: com concordância, quando a diferença de pesos era inferior a $15 \%$, com discordância leve, quando a diferença estava entre 15 e $25 \%$, e com discordância grave, quando a diferença era superior a $25 \%{ }^{5}$. Foram considerados de baixo peso os RN que apresentavam peso ao nascer inferior a $2.500 \mathrm{~g}^{13}$. Esses RN foram distribuídos de acordo com o índice de Apgar de primeiro e quinto minuto, em menor e maior ou igual a 7 , e avaliados nesses dois tempos ${ }^{14}$. A duração das gestações foi estimada com base na data da última menstruação e foi confirmada pelo exame ecográfico. Esse exame estimou, também, o tempo de gestação quando a data menstrual era desconhecida.

Quando a duração da gravidez era inferior a 37 semanas, os RN foram classificados como prétermo. A classificação do Comitê de Normas da OMS (1972), adotada no Serviço de Neonatologia do Centro de Ciências Médicas e Biológicas da Pontifícia Universidade Católica de São Paulo (CCMB/ PUC-SP), foi utilizada para distribuir os $\mathrm{RN}$ de acordo com a idade gestacional no parto e peso ao nascimento. O tempo médio de internação dos RN no berçário, em dias, foi calculado para os dois gêmeos, em separado. O coeficiente de mortalidade perinatal I de gêmeos foi calculado pelos critérios da OMS/FIGO $(1977)^{15}$, para determinada região e por ano: nascidos mortos + óbitos de 0-7 dias/total de nascimentos x 1000 .

Utilizou-se a análise de variância por postos de Kruskal-Wallis, com a finalidade de comparar as três classes de diferença de pesos entre gêmeos em relação à idade materna média e ao tempo de internação dos recém-nascidos no berçário. Quando mostrou diferença significante, a análise foi complementada pelo teste de comparações múltiplas (Hollander). Para as demais características estudadas, utilizou-se o teste das proporções independentes (Blackwell), com o objetivo de comparar as porcentagens de diferença de pesos entre gêmeos. $O$ teste foi aplicado para os primeiros e segundos gêmeos, em separado, e na comparação entre ambos. O nível de significância adotado foi de $10 \%$, porque, para o pequeno tamanho amostral, não havia cálculo algum que indicasse quantas amostras tomar, em outros niveis de significância.

Este trabalho foi aprovado pelos Comitês de Ética em Pesquisa do CCMB/PUC-SP e da Faculdade de Medicina de Botucatu - UNESP em 2 de dezembro de 1999.

\section{Resultados}

Das 89 gestações múltiplas, $62(69,7 \%)$ foram de gêmeos com concordância ponderal, 17 (19,1\%) com discordância leve e $10(11,2 \%)$ com discordância grave. O coeficiente de mortalidade perinatal I, para a população estudada, foi de $67,4 \%$ o. Na classe com discordância leve ocorreu menor número de cesáreas $(41,2 \%)$, ao passo que na com discordância grave predominou a multiparidade (80\%). A interrupção prematura da gestação ocorreu em 48 casos (53,9\%), sendo 7 deles na classe com discordância grave $(70 \%)$ (Tabela 1$)$.

Tabela 1 - Características maternas das 89 gestações de gêmeos, distribuídas nas três classes de diferença de pesos (CDP).

\begin{tabular}{|c|c|c|c|c|c|c|c|c|c|}
\hline \multirow{2}{*}{ CDP } & \multicolumn{2}{|c|}{$\begin{array}{l}\text { Gestações } \\
\text { gemelares }\end{array}$} & \multirow{2}{*}{$\begin{array}{l}\text { Idade materna } \\
\text { média (anos) }\end{array}$} & \multicolumn{2}{|c|}{ Multíparas } & \multicolumn{2}{|c|}{ Cesáreas } & \multicolumn{2}{|c|}{$\begin{array}{c}\text { IGP } \\
<37 \text { sem. }\end{array}$} \\
\hline & $\mathrm{n}$ & $\%$ & & $\mathrm{n}$ & $\%$ & $\mathrm{n}$ & $\%$ & $\mathrm{n}$ & $\%$ \\
\hline Concordância & 62 & 69,7 & $26,5 a$ & 47 & $75,8 a$ & 37 & $51,4 a$ & 32 & $51,6 a$ \\
\hline Discordância leve & 17 & 19,1 & $29,8 a$ & 13 & $76,5 a$ & 7 & $41,2 b$ & 9 & $52,9 a b$ \\
\hline Discordância grave & 10 & 11,2 & $24,3 a$ & 8 & $80,0 \mathrm{~b}$ & 7 & $70,0 \mathrm{a}$ & 7 & $70,0 \mathrm{~b}$ \\
\hline Média & & & 26,8 & & 76,4 & .. & 57,3 & & 53,9 \\
\hline
\end{tabular}

Média e proporções seguidas de letras iguais, na comparação entre as três classes de diferença de peso, não diferem entre si para $10 \%$ de probabilidade.

$\mathrm{Na}$ comparação entre os primeiros gêmeos, nas três classes de diferença de pesos, eles não diferiram significativamente com relação à incidência de baixo peso ao nascer, índice de Apgar menor que 7 no primeiro e quinto minuto e coeficiente de mortalidade perinatal I. O tempo médio de internação no berçário foi maior nos gêmeos com discordância leve (4,6 dias) e grave (7,3 dias) (Tabela 2$)$. 
Tabela 2 - Características comparativas do primeiro e segundo gêmeo nas 89 gestações, nas três classes de diferença de pesos.

\begin{tabular}{|c|c|c|c|c|c|c|c|c|c|c|}
\hline \multirow{3}{*}{ CDP } & & & \multicolumn{4}{|c|}{ Apgar < $7 \quad(\%)$} & \multirow{2}{*}{\multicolumn{2}{|c|}{$\begin{array}{l}\text { Tempo médio de } \\
\text { internação (dias) }\end{array}$}} & \multirow{2}{*}{\multicolumn{2}{|c|}{ CMPN I (\%) }} \\
\hline & \multicolumn{2}{|c|}{ Baixo peso (\%) } & \multicolumn{2}{|c|}{$1^{\circ}$ minuto } & \multicolumn{2}{|c|}{$5^{\circ}$ minuto } & & & & \\
\hline & $1^{0}$ & $2^{\circ}$ & $1^{0}$ & $2^{0}$ & $1^{0}$ & $2^{\circ}$ & $1^{0}$ & $2^{0}$ & $1^{0}$ & $2^{0}$ \\
\hline Concordância & $62,9 a$ & $66,1 a$ & $25,8 a$ & $20,9 a$ & $6,4 a$ & $6,4 a$ & $3,7 a$ & $3,7 a$ & $22,4 a$ & $16,8 a$ \\
\hline Discordância leve & $76,5 a$ & $70,6 a$ & $17,6 a$ & $41,1 \mathrm{~b}$ & 0 & $11,7 \mathrm{~b}$ & $4,6 a$ & $6,0 a$ & 0 & $16,8 a$ \\
\hline Discordância grave & $80,0 a$ & $90,0 \mathrm{a}$ & $20,0 a$ & $30,0 \mathrm{a}$ & $10,0 \mathrm{a}$ & $20,0 \mathrm{a}$ & $7,3 a$ & $8,7 a$ & $5,6 a$ & $5,6 a$ \\
\hline Média & 67,4 & 69,6 & 23,6 & 25,8 & 5,6 & 9,0 & 5,2 & 6,1 & 28,0 & 39,2 \\
\hline
\end{tabular}

Média e proporções seguidas de letras iguais, na comparação entre as três CDP, não diferem entre si para $10 \%$ de probabilidade.

CMPNI - coeficiente de mortalidade perinatal I.

Na comparação entre os segundos gêmeos, nas três classes de diferença de pesos, a maior incidência de baixo peso ao nascer (90\%) ocorreu na classe com discordância grave. O tempo médio de internação no berçário foi maior nas classes com discordância leve (6 dias) e grave (8,7 dias). $\mathrm{Na}$ classe com discordância leve, foi maior a incidência de Apgar menor que 7 no primeiro minuto $(41,1 \%)$ (Tabela 2).

$\mathrm{Na}$ comparação dos resultados entre o primeiro e o segundo gêmeo, observamos que apenas o segundo gêmeo, da classe com discordância leve, apresentou maior incidência de Apgar menor que 7 , no primeiro e quinto minuto $(41,1 \mathrm{e}$ $11,7 \%$ ) (Tabela 2).

\section{Discussão}

A falha ou diminuição no crescimento intrauterino pode ocorrer com um ou ambos os gêmeos do par. Não há regra que explique essa ocorrência e, tampouco, quais valores de diferenças são relevantes na correlação com os resultados perinatal e neonatal ${ }^{4}$. É muito analisada na literatura a estimativa dessas disparidades ponderais, em peso absoluto ou em percentual, e as conseqüências para os conceptos ${ }^{6,7}$.

Nessa estimativa, parece consenso utilizarse o maior gêmeo como $100 \%$ e considerar discordância de crescimento quando os pesos diferem de 20 a $25 \%{ }^{6}$. Blickstein et al. ${ }^{6}$, encontraram diferenças variando de 10 a $36 \%$. Denominaram discordância a diferença igual ou superior a $15 \%$ e concordância, os percentuais menores. Subdividiram a discordância em leve, quando situada entre 15 e $25 \%$, e grave, quando acima dos $25 \%^{5}$, critério esse também utilizado por outros auto$\operatorname{res}^{4,7}$. Valores de 10 e $20 \%$ também já foram utilizados para caracterizar tais discordâncias ${ }^{10}$.

A mesma fórmula foi empregada para calcular o percentual de diferença de pesos no grupo em estudo e caracterizar as três classes de diferença ponderal empregadas no trabalho. Tal me- todologia foi escolhida por ser a mais utilizada na literatura, permitindo, assim, maior número de parâmetros para comparar os resultados.

A gravidez gemelar é considerada de risco pela elevada taxa de mortalidade perinatal que, em alguns países, é 4 a 5 vezes maior que a da gravidez única ${ }^{15}$. A causa mais importante de mortalidade é a prematuridade e suas complicações ${ }^{16}$. A taxa de prematuridade observada $(53,9 \%)$ é muito alta, pois os relatos da literatura oscilam entre 25 e $30 \%{ }^{17}$. O coeficiente de mortalidade perinatal I $(67,4 \%$ o) é cerca de 10 vezes maior que o observado nas gestações de feto único, no HRCS.

Além da prematuridade, deve-se enfatizar a incidência de baixo peso ao nascer $(68,5 \%)$, similar à literatura, que mostra mais da metade dos gêmeos nascendo com peso inferior a $2.500 \mathrm{~g}^{8,18}$. Os dois fatores adversos, que contribuíram para isso, são a discordância de pesos superior a $25 \%$ e os fatores demográficos de alto risco, como a desnutrição, responsáveis pelo nascimento de crianças com peso em torno de $2.000 \mathrm{~g}^{19}$.

A taxa de concordância de pesos entre pares de gêmeos é de 15 a $29 \%$, dependendo do critério utilizado $^{3,5}$. Utilizando $15 \%$ como referência, critério que julgamos adequado pelo baixo número amostral, nossos resultados mostraram a maioria dos gêmeos na classe com concordância. A discordância leve ocorreu em $19,1 \%$ e a grave em $11,2 \%$, utilizando-se o critério de Blickstein et al. ${ }^{6}$, que citam a discordância leve variando de 19 a $23 \%$. Utilizando-se percentual de $25 \%$, há relatos de $8,2 \%$ de discordância ${ }^{10}$.

Algumas características maternas podem influenciar a ocorrência de discordância de pesos entre pares de gêmeos, como a idade e a paridade. Nascimentos gemelares são mais comuns em mulheres entre 30 e 40 anos de idade ${ }^{20}$; no período de 1974 a 1994, por exemplo, a taxa de gemelidade nas mulheres norte-americanas com mais de $30 \operatorname{anos}^{21}$ foi duas vezes maior. No nosso trabalho, observamos grávidas com idade variando de 15 a 42 anos (média de 26,8), à semelhança de Blickstein et al. ${ }^{6}$, com 27,7 anos, Cheung et al. ${ }^{4}$, com 27,8 anos, e Yalçin et al. ${ }^{7}$, com 25,8 anos. 
As mulheres da classe com discordância grave eram mais jovens (24,3 anos) e as da classe com discordância leve mais idosas (29,8 anos), não havendo diferença significante entre elas.

A multiparidade predominou entre as mães, sendo maior na classe com discordância grave de peso. Observamos mulheres com 1 a 9 partos (média de 3,1), à semelhança de Blickstein et al. ${ }^{6}$, com 1 a 8 (média de 2,4), que não verificaram diferença entre pares concordantes e discordantes. Yalçin et al. ${ }^{7}$ citaram mulheres com 1 a 5 partos (média de 2,2).

A Disciplina de Obstetrícia do CCMB/PUCSP, que coordena a Maternidade do HRCS, utiliza o conceito de parto como a interrupção da gravidez com mais de 20 semanas, critério que foi empregado no estudo. A freqüência de cesáreas $(57,3 \%)$ foi elevada nas três classes de diferença de pesos e menor na classe com discordância leve. Houve, entretanto, tendência maior de ocorrência de cesáreas na discordância grave de pesos $(70 \%)$. Os resultados assemelham-se aos da literatura, pela incidência de altas taxas de cesáreas $^{7,22}$, mas distinguem-se na comparação estatística entre as classes, pois a proporção é maior na com discordância leve ${ }^{4}$. Taxas elevadas de cesáreas, além de não melhorar o prognóstico neonatal ${ }^{22}$, agravam o materno ${ }^{23}$.

Num estudo com 1075 placentas de gestações gemelares, 284 estavam associadas a gêmeos com pesos discordantes. Tais placentas apresentavam anomalias no cordão umbilical, com pequeno volume de cório frondoso e diminuição do número de vasos coriônicos ${ }^{1}$, sugerindo influência da perda de desenvolvimento placentário para um dos gêmeos; porém, a causa básica é desconhecida. Em decorrência desses conhecimentos, é de se esperar que o resultado perinatal seja melhor na concordância de pesos, intermediário na discordância leve e pior na discordância grave.

O tamanho amostral pequeno, com apenas 178 gêmeos, impediu a comprovação estatística de todos os parâmetros. Entretanto, a análise dos percentuais evidencia que na classe com concordância houve menor incidência de baixo peso ao nascer e de prematuridade, bem como menor tempo médio de internação no berçário. Tal parâmetro é utilizado como indice para avaliar o bem-estar neonatal ${ }^{6}$. A classe com discordância leve mostrou comportamento intermediário dessas três variáveis. Na discordância grave observamos maior incidência de baixo peso ao nascer, maior tempo médio de internação no berçário e maior percentual de indice de Apgar inferior a 7 no quinto minuto. Esses achados, apesar de não evidenciarem diferenças estatisticamente significantes, são importantes na orientação do seguimento pré-natal.
Na classe com discordância grave, 7 pares de gêmeos nasceram antes do termo $(70,0 \%), 8$ primeiros gêmeos $(80,0 \%)$ e 9 segundos gêmeos $(90 \%)$ apresentaram baixo peso e houve 2 natimortos (1 primeiro e 1 segundo gêmeo), o que revelou indices absolutos muito altos. Ainda, a incidência de baixo peso ao nascer foi estatisticamente superior para o segundo gêmeo e muito próxima da significância estatística para o primeiro gêmeo. A amostra com 10 casos é pouco expressiva, mas suficiente para causar preocupação em se diagnosticarem previamente esses pares de alto risco, visando à melhoria de seus prognósti$\cos ^{23}$.

Nasceram 62 segundos gêmeos $(69,6 \%)$ com baixo peso, dados similares aos da literatura ${ }^{8,18}$. Alguns trabalhos têm mostrado pior resultado perinatal para o segundo gêmeo ${ }^{16,24}$. A comparação feita entre o primeiro e o segundo gêmeo, nas três classes de diferença de pesos, mostrou, apenas, que os segundos gêmeos, no grupo com discordância leve, tiveram maior incidência de Apgar menor que 7 no primeiro e quinto minutos. Provavelmente, os cuidados antenatais e a atenção intraparto reduziram as diferenças da morbidade perinatal entre eles.

A semelhança com os dados da literatura ${ }^{6,7}$, que associam a maior permanência hospitalar ao risco perinatal elevado, devido à maior morbilidade e mortalidade ${ }^{24}$, foi observada, embora não haja consenso sobre essa correlação ${ }^{10}$. A permanência mais longa pode ser explicada pela necessidade de maior manipulação de neonatos hipóxicos e prétermo.

A falta de consenso se reflete nos trabalhos sobre resultados perinatais confrontando o tipo de parto, a análise dos tempos de desprendimento no parto, o acompanhamento neuro-psico-motor dos neonatos, o diagnóstico preditivo da discordância de pesos, a prevenção da prematuridade e a padronização dos pesos baseada na corionicidade placentária, os quais têm sido objeto de estudos recentes $^{12,24,25}$.

\section{ABSTRACT}

Purpose: to study the influence of weight differences between twins on the perinatal results.

Methods: the twin deliveries at the Maternity of the Hospital Regional de Clinicas de Sorocaba, SP, were retrospectively analyzed from July 1997 to June 1998. The samples were 89 mothers and their twins, divided into three classes of newborn weight differences, as follows: concordant $(<15 \%)$, mild discordance (15 to 25\%) and severe discordance (>25\%). The independent variables analyzed were these three classes 
and the dependent variables were low weight at birth, Apgar index less than 7 at the first and fifth minute, premature delivery, time of permanence of the newborn in the nursery, and perinatal mortality coefficient I. Statistical analysis was performed using Kruskal-Wallis test, completed by Hollander test, and the Blackwell test.

Results: the number of pregnancies $(62,17$ and 10) and premature deliveries (32, 9 and 7) were observed respectively in the three classes. For the first and second twins we observed: low weight at birth (39/41, 13/12 and 8/9), Apgar index less than 7 at the first minute (16/13, 3/7 and 2/3), Apgar index at the fifth minute (4/4, 0/2 and 1/2), time (in days) of permanence of the newborn in the nursery (3.7/3.7, 4.6/6.0 and 7.3/8.7) and perinatal mortality coefficient I (22.4/16.8, 0/16.8 and 5.6/5.6).

Conclusions: the incidence of weight discordance between twins was 30.3\%, 19.1\% being mild discordance and $11.2 \%$ severe discordance. There was a tendency to a progressive aggravation of perinatal results considering the degree of discordance of the classes (concordant $<$ mild discordance $<$ severe discordance).

KEY WORDS: Twins. Discordant twins. Perinatal morbidity and mortality.

\section{Referências}

1. Baldwin VJ. Morbidity in twins. In: Baldwin VJ, editor. Pathology of Multiple Pregnancy. $1^{\text {st }}$ ed. New York: Springer-Verlag; 1994. p.133-67.

2. Bronsteen R, Goyert G, Bottoms S. Classification of twins and neonatal morbidity. Obstet Gynecol 1989; 74:98-101.

3. Rodis JF, Vintzileos AM, Campbell WA, Nochimson DJ. Intrauterine fetal growth in discordant twin gestations. J Ultrasound Med 1990; 9:443-8.

4. Cheung VY, Bocking AD, Dasilva OP. Preterm discordant twins: what birth weight difference is significant? Am J Obstet Gynecol 1995; 172:955-9.

5. Blickstein I, Lancet M. The growth discordant twin. Obstet Gynecol Surv 1988; 43:509-15.

6. Blickstein I, Shohan-Schwartz Z, Lancet M, Borenstein R. Characterization of the growthdiscordant twin. Obstet Gynecol 1987; 70:11-5.

7. Yalçin HR, Zorlu CG, Lembet A, Özden S, Gökmen $O$. The significance of birth weight difference in discordant twins: a level to standardize? Acta Obstet Gynecol Scand 1998; 77:28-31.

8. Ho SK, Wu PYH. Perinatal factors and neonatal morbidity in twin pregnancy. Am J Obstet Gynecol 1975; 122: 979-87.

9. Bleker OP, Oosting H. Term and postterm twin gestation. Placental cause of perinatal mortality. J Reprod Med 1997; 42:715-8.
10.Guerzet EA. Desfecho neonatal em gêmeos com pesos discordantes na Maternidade Pró-Matre de Vitória [dissertação]. São Paulo: Universidade Federal de São Paulo; 1999.

11.Hsieh TT, Chang TC, Chiu TH, Hsu JJ, Chao A. Growth discordancy, birth weight, and neonatal adverse events in third trimester twin gestations. Gynecol Obstet Invest 1994; 38:36-40.

12.Petterson B, Blair E, Watson L, Stanley F. Adverse outcome after multiple pregnancy. Baillieres Clin Obstet Gynaecol 1998; 12:1-17.

13.Amon E. Limit of fetal viability: obstetric considerations regarding the management and delivery of the extremely premature baby in high risk pregnancy. Obstet Gynecol Clin North Am 1988; 15:321-38.

14.Usher R. Extreme prematurity. In: Avery GB, editor. Neonatology, Pathophysiology and Management of the Newborn. $3^{\text {rd }}$ ed. Philadelphia: JB Lippincott; 1987 ; p.264.

15. Cecatti JG, de Aquino MMA. Mortalidade perinatal. In: Neme B, editor. Obstetrícia Básica. $2^{\mathrm{a}}$ ed. São Paulo: Sarvier; 2000. p.1016-21.

16. Owen P, Patel NB. Epidemiology of multiple pregnacy. In: Ward RH, Whittle M, editors. Multiple Pregnancy. London: RCOG Press; 1995. p.1-13.

17.Sassoon DA, Castro LC, Davis JL, Hobel CJ. Perinatal outcome in triplet versus twin gestations. Obstet Gynecol 1990; 75:817-20.

18.Da Silva JCFG, Milanez HMBPM. Gestação Múltipla. In: Neme B, editor. Obstetrícia Básica. $2^{a}$ ed. São Paulo: Sarvier; 2000. p.263-75.

19. Fowler MG, Kleinman JC, Kiely JL, Kessel SS. Double jeopardy: twin infant mortality in the United States, 1983 and 1984. Am J Obstet Gynecol 1991; 165:15-22.

20.Kiely JL. What is the population-based risk of preterm birth among twins and other multiples? Clin Obstet Gynecol 1998; 41:3-11.

21.Luke B. What is the influence of maternal weight gain on the fetal growth of twins? Clin Obstet Gynecol 1998; 41:57-64.

22. Rydhström H. Prognosis for twins discordant in birth weight of $1.0 \mathrm{~kg}$ or more: the impact of cesarean section. J Perinat Med 1990; 18:31-7.

23.Blickstein I, Manor M, Levi R, Goldchmit R. Is intertwin birth weight discordance predictable? Gynecol Obstet Invest 1996; 42:105-8.

24.el-Jallad MF, Abu-Heija AT, Ziadeh S, Obeidat A. Is the second-born twin at high risk? Clin Exp Obstet Gynecol 1997; 24:226-7.

25.Ananth CV, Vintzileos AM, Shen-Schwarz S, Smulian JC, Lai YL. Standards of birth weight in twin gestations stratified by placental chorionicity. Obstet Gynecol 1998; 91:917-24.

Recebido em: 7/2/2002 Aceito com modificações em: 15/7/2002 